\title{
Ударная электромагнитная волна большой амплитуды в нелинейной линии передачи на основе распределенного полупроводникового диода
}

\author{
(C) A.C. Кюрегян
}

Всероссийский электротехнический институт им. В.И. Ленина, 111250 Москва, Россия

E-mail: semlab@yandex.ru

(Получена 23 октября 2017 г.

Принята к печати 29 сентября 2018 г.

Принята к публикации 29 октября 2018 г.)

Получено аналитическое решение задачи о возникновении и распространении ударной электромагнитной волны большой амплитуды в полосковой линии передачи на основе распределенного полупроводникового диода, которое корректно учитывает ее нелинейность, диссипацию и временную дисперсию. Результаты теории использованы для оценки параметров линии передачи как обострителя фронта импульса напряжения, подаваемого на вход линии.

DOI: $10.21883 /$ FTP.2019.04.47452.8752

\section{1. Введение}

Давно известно, что в нелинейных линиях передачи (ЛП) при определенных условиях возникают ударные электромагнитные волны, которые используются, в частности, для формирования импульсов напряжения с крутыми фронтами [1-6]. Нелинейными элементами могут быть распределенные вдоль линии индуктивность $L$, зависящая от тока $I$ при использовании ферритов, и(или) емкость $C$, зависящая от напряжения $U$ при использовании ферроэлектриков, полупроводниковых МДПструктур, диодов с барьером Шоттки или с $p^{+}-n$-переходом. Именно этот последний вариант ЛП теоретически исследован в настоящей работе применительно к проблеме обострения импульсов напряжения большой амплитуды.

Такую ЛП можно представить в виде множества последовательно соединенных ячеек, эквивалентная схема которых изображена на рис. 1, $a$. Распространение по ней электромагнитной волны вдоль оси $z$ описывается при известных [7] условиях системой телеграфных уравнений:

$$
\frac{\partial U}{\partial z}+L \frac{\partial I}{\partial t}+R_{L} I=0, \quad \frac{\partial I}{\partial z}+\frac{\partial Q}{\partial t}+G U=0,
$$

где $I=I(t, z)$ - ток вдоль линии, $L$ и $R_{L}-$ постоянные погонные индуктивность и сопротивление линии, $G^{-1}$ - погонное сопротивление утечки. Нелинейность соотношения между напряжением $U=U(t, z)$ и погонной плотностью заряда $Q=Q(t, z)$ линии является причиной возникновения ударной волны. Среди использованных ранее соотношений наиболее общее и подходящее для нашего случая было предложено авторами работы [8]. Его можно представить в виде двух равенств:

$$
U=Q / C_{w}(Q)+U_{n}, \quad U_{n}=R_{n} \partial\left(Q-C_{n} U_{n}\right) / \partial t,
$$

где $C_{w}$ - барьерная емкость диода, $U_{n}-$ падение напряжения на емкости $C_{n}$ и сопротивлении $R_{n}-$ нейтральной области $n$-слоя диода (см. рис. $1, b)$. Авторы остальных известных нам работ [1,2,9-13] либо использовали неподходящие для нашего случая зависимости $C_{w}(Q)$, либо пренебрегали величинами $C_{n}$ и(или) $R_{n}$, а те, кто их учитывал (в том числе и авторы работы [8]), полагали $C_{n}$ и $R_{n}$ постоянными. На самом деле при большой амплитуде волны величины $R_{n}$ и $C_{n}$ явно зависят от времени из-за уменьшения толщины $(d-w)$ нейтральной области $n$-слоя, а $R_{n}$ зависит еще и от плотности тока $J=\partial Q / \partial t$, протекающего поперек ЛП, вследствие уменьшения подвижности электронов с ростом напряженности электрического поля.

Способ учета этих особенностей, позволяющий корректно описать нелинейность, диссипацию и временну́ю дисперсию активного $n$-слоя диода, изложен в разд. 2 настоящей статьи. В разд. 3 дано аналитическое описание реальной структуры фронта стационарной ударной волны в ЛП на основе распределенного диода, в разд. 4 описаны процессы формирования и затухания ударной волны при подаче на вход ЛП относительно медленно нарастающего напряжения, а в Заключение дана оценка параметров ЛП как обострителя фронта импульса напряжения.

\section{2. Нелинейность и временна́я дисперсия диодов}

Рассмотрим полосковую линию передачи, состоящую из протяженного вдоль оси $z$ обратно смещенного $p^{+}-n-n^{+}$-диода, поперечное сечение которого схематически изображено на рис. $1, b$. Будем считать, что: $-n^{+}$-подложка толщиной $d_{s}$ однородно легирована донорами с концентрацией $N_{s}$,

- активная $n$-область толщиной $d$ однородно легирована донорами с концентрацией $N \ll N_{s}$,

$-p^{+}$-слой (на рисунке не показан) толщиной $d_{p} \ll d$ легирован акцепторами с концентрацией $N_{p} \gg N$, 
Таблица 1. Подгоночные параметры, входящие в формулы (5) и (7), для $\mathrm{Si}$ и $4 H-\mathrm{SiC}$

\begin{tabular}{c|c|c|c|c|c|c}
\hline Параметр & $\begin{array}{c}v_{e}, \\
\mathrm{cм} / \mathrm{M \kappa c}\end{array}$ & $\begin{array}{c}E_{e}, \\
\mathrm{\kappa B} / \mathrm{cm}\end{array}$ & $\begin{array}{c}E_{1}, \\
\text { кВ/см }\end{array}$ & $\begin{array}{c}E_{2}, \\
\text { кВ/см }\end{array}$ & $\begin{array}{c}E_{i}, \\
\mathrm{MB} / \mathrm{cm}\end{array}$ & $\begin{array}{c}U_{i}, \\
\mathrm{~B}\end{array}$ \\
\hline $\mathrm{Si}$ & 10.0 & 7.1 & $\infty$ & $\infty$ & 1.43 & 1.83 \\
$4 H-\mathrm{SiC}$ & 18.5 & 21 & 900 & 550 & 15.9 & 1.15
\end{tabular}

- ширина полоски $l$ и толщина фронта $\delta_{f}$ ударной волны, распространяющейся по линии, много больше $\left(d+d_{s}\right)$,

- проводимость $G$ истощенной области полупроводника $(0<x<w)$ пренебрежимо мала.

Тогда поперечное электрическое поле $E$ в $n$-области практически не зависит от $y$, а вдоль оси $x$ распределено так, как изображено на рис. 1, c. Разность потенциалов между электродами $U(t, z)$, включающая и контактную разность потенциалов, связана с напряженностями $E_{0}(t, z), E_{n}(t, z), E_{s}(t, z)$ в плоскостях $x=0, x=d$, $x=d+d_{s}$ и с толщиной истощенной области $w(t, z)$ очевидными соотношениями:

$$
\begin{gathered}
U=\left(E_{0}-E_{n}\right) \frac{w}{2}+E_{n} d+E_{s} d_{s}, \\
E_{0}=\frac{e}{\varepsilon} N w+E_{n},
\end{gathered}
$$

где $\varepsilon$ - диэлектрическая проницаемость полупроводника, $e-$ элементарный заряд. Далее для определенности мы будем считать, что в статическом случае (т.е. при $\left.E_{n}=E_{s}=0\right) w=d$, и $E_{0}=E_{B}=e N d / \varepsilon$ при $U=U_{B}$, где $U_{B}$ - напряжение лавинного пробоя, связанное с $d$ и $E_{B}$ соотношениями

$$
U_{B}=E_{B} d / 2, \quad E_{B}=E_{i} / \ln \left(U_{B} / U_{i}\right)
$$

$E_{i}$ и $U_{i}$ - подгоночные параметры, приведенные в табл. 1 для $\mathrm{Si}[14]$ и $4 H-\mathrm{SiC}[15]$.

Электрические поля $E_{n}, E_{s}$ в областях $w<x<d$, $d<x<d+d_{s}$ не зависят от $x$ и обусловлены протекающим через них током с линейной плотностью

$$
\begin{aligned}
J & =\frac{\partial Q}{\partial t}=l \varepsilon \frac{\partial E_{0}}{\partial t}=l\left[e N v\left(E_{n}\right)+\varepsilon \frac{\partial E_{n}}{\partial t}\right] \\
& =l e N_{s} \mu_{e s} E_{s}
\end{aligned}
$$

где $Q(t, z)=l \varepsilon E_{0}(t, z)$ - погонная плотность заряда в плоскости $x=0, v(E)$ - дрейфовая скорость электронов, для которой мы будем использовать аппроксимацию

$$
v(E)=v_{e} \frac{1+\left(E / E_{1}\right)^{2}}{E_{e} / E+1+\left(E / E_{2}\right)^{2}},
$$

$v_{e}=\mu_{e} E_{e}, \mu_{e}$ и $\mu_{e s}$ - низкополевые подвижности электронов в $n$-области и подложке. Она хорошо описывает реальные зависимости $v(E)$ в $\mathrm{Si}[14]$ (где скорость практически постоянна в сильном поле) и $4 \mathrm{H}-\mathrm{SiC}$ [15] (где скорость уменьшается с ростом $E$ при $E>200$ кВ.см) при значениях подгоночных параметров, приведенных в табл. 1.

Первое слагаемое в квадратных скобках формулы (6) соответствует току проводимости через сопротивление $R_{n}$, а второе - току смещения через емкость $C_{n}$. Так как обычно $N_{s} \gg N$, в подложке можно пренебречь током смещения и снижением подвижности в поле $E_{s} \ll E_{e}$. Из (4) и (6) следует, что

$$
\partial w / \partial t=v\left(E_{n}\right)
$$

т.е. граница $x=w$ между истощенной и нейтральной областями $n$-слоя движется со скоростью $v\left(E_{n}\right)$, изменяя тем самым эффективные значения $R_{n}$ и $C_{w, n}$. Формулы
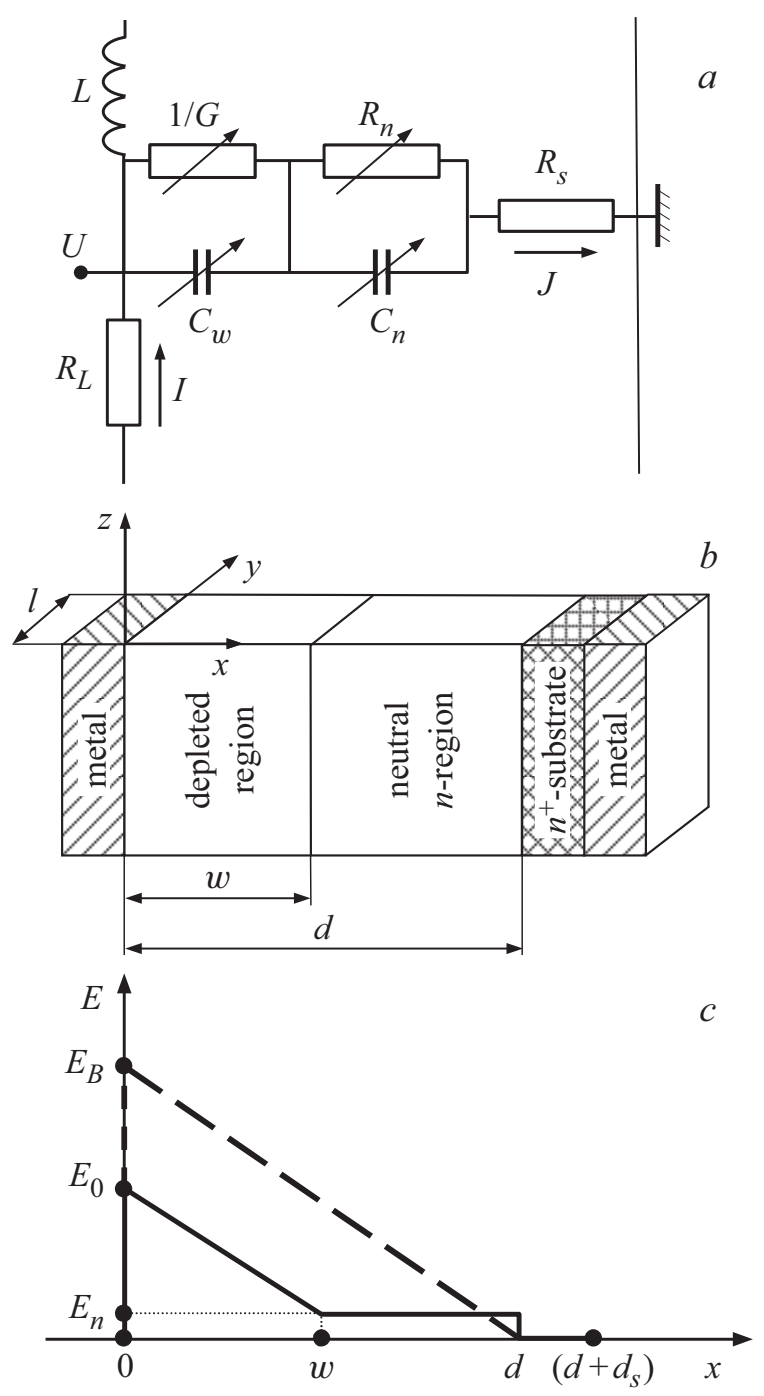

Рис. 1. Эквивалентная схема ячейки нелинейной линии передачи $(a)$, поперечное сечение полоскового диода $(b)$ и распределение электрического поля в $p^{+}-n-n^{+}$-структуре $(c)$. $C_{w}$ и $G-$ емкость и проводимость истощенной области полупроводника $(0<x<w), C_{n}$ и $R_{n}-$ емкость и сопротивление нейтральной области $n$-слоя $(w<x<d), R_{s}-$ сопротивление подложки $(d<x<d s)$. Тонкий $p^{+}$-слой не показан. 
(3)-(6) и (8) задают в неявном виде соотношение между $U$ и $Q$ и являются аналогом равенств (2), однако только они корректно и исчерпывающе описывают особенности нелинейности, диссипации и временно́й дисперсии активного $n$-слоя диода.

\section{3. Стационарная ударная волна}

Стационарная ударная волна, строго говоря, может существовать только в бесконечно длинной ЛП с пренебрежимо малыми сопротивлением $R_{L}$ и проводимостью $G$. В этом случае в системе координат $\tilde{z}=z-u_{f} t$, движущейся вместе с фронтом с постоянной скоростью $u_{f}$, уравнения (1) принимают вид

$$
\frac{\partial U}{\partial \tilde{z}}-L u_{f} \frac{\partial I}{\partial \tilde{z}}=0, \quad \frac{\partial I}{\partial \tilde{z}}-u_{f} \frac{\partial Q}{\partial \tilde{z}}=0 .
$$

Интегрирование (9), с граничными условиями

$$
U( \pm \infty)=U_{ \pm}, \quad Q( \pm \infty)=Q_{ \pm}, \quad I( \pm \infty)=0
$$

приводит к соотношениям

$$
Q(\tilde{z})-Q_{+}=\bar{C}\left[U(\tilde{z})-U_{+}\right], \quad U(\tilde{z})-U_{+}=L u_{f} I(\tilde{z}),
$$

где эффективная погонная емкость

$$
\bar{C}=\frac{Q_{-}-Q_{+}}{U_{-}-U_{+}}=\frac{\varepsilon l}{\bar{w}},
$$

$\bar{w}=\left(w_{+}+w_{-}\right) / 2, \quad w_{ \pm}=2 U_{ \pm} / E_{ \pm}, \quad E_{ \pm}=E_{B} \sqrt{U_{ \pm} / U_{B}}$ $=Q_{ \pm} / l \varepsilon$.

Далее мы будем считать, что параметры подложки $N_{s}$, $d_{s}$ таковы, что выполняются два сильных неравенства:

$$
E_{s} d_{s} \ll E_{n} d \quad \text { и } \quad d_{s} \ll h_{s}=2 \sqrt{t_{f} / \mu_{0} \sigma_{s}},
$$

где $h_{s}-$ глубина проникновения магнитного поля в подложку с проводимостью $\sigma_{s}=e \mu_{e s} N_{s}$ за время $t_{f}=\delta_{f} / u_{f}$ нарастания тока на фронте волны, $\mu_{0}-$ магнитная постоянная. В этом случае диссипация в подложке пренебрежимо мала, погонная индуктивность линии $L=\mu_{0}\left(d+d_{s}\right) / l$ и скорость фронта

$$
u_{f}=(L \bar{C})^{-1 / 2}=c_{s} \sqrt{\frac{\bar{w}}{d+d_{s}}}
$$

могут быть значительно меньше скорости света $c_{s}=1 / \sqrt{\varepsilon \mu_{0}}$ в полупроводнике [16-21]. Волновое сопротивление такой линии равно

$$
Z=\frac{U(\tilde{z})-U_{+}}{I(\tilde{z})}=\sqrt{\frac{L}{\bar{C}}}=Z_{s} \frac{\sqrt{\bar{w}\left(d+d_{s}\right)}}{l},
$$

где $Z_{s}=\sqrt{\mu_{0} / \varepsilon} \approx 110$ Ом в кремнии.

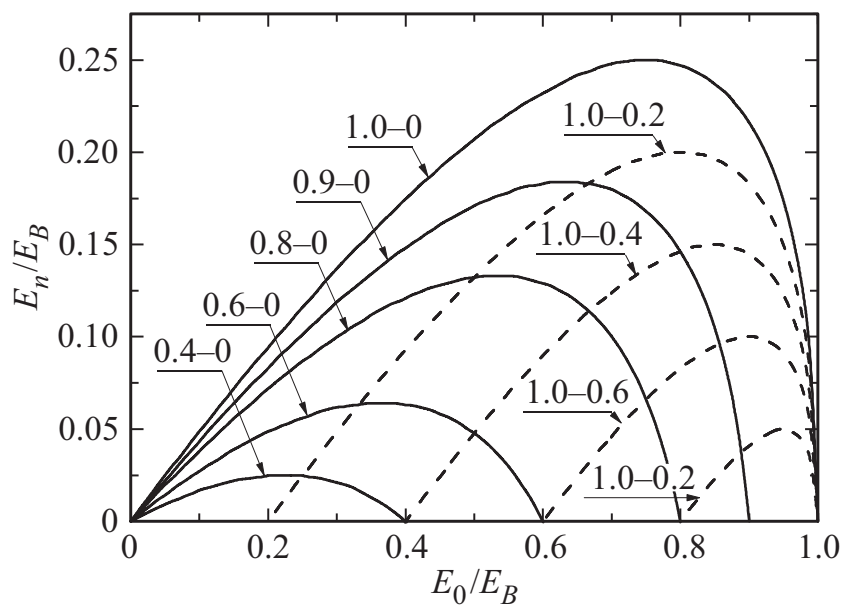

Рис. 2. Графики функции $E_{n}\left(E_{0}\right)$, рассчитанные по формуле (16). Числа у кривых указывают значения отношений $E_{-} / E_{B}$ (первое) и $E_{+} / E_{B}$ (второе).

Используя (3) без малого слагаемого $E_{s} d_{s}$ вместе с первым из равенств (11), легко показать, что при этих условиях

$$
\begin{array}{r}
E_{n}\left(E_{0}\right)=E_{0}-E_{B}+\sqrt{\left(E_{B}-E_{0}\right)^{2}+\left(E_{-}-E_{0}\right)\left(E_{0}-E_{+}\right)}, \\
U\left(E_{0}\right)=U_{B} E_{B}^{-2}\left[E_{0}\left(E_{+}+E_{-}\right)-E_{+} E_{-}\right] .
\end{array}
$$

Как и следовало ожидать, при $E_{0} \rightarrow E_{ \pm}$(т.е. вдали от фронта, где $J \rightarrow 0) U_{0} \rightarrow U_{ \pm}$, а напряженность поля $E_{n} \rightarrow 0$ и достигает максимума

$$
\hat{E}_{n}=\frac{\bar{E}^{2}}{2\left(E_{B}-\bar{E}\right)}
$$

на фронте при

$$
E_{0}=\hat{E}_{0}=\frac{E_{B} E_{+}+E_{B} E_{-}-E_{+} E_{-}-\bar{E}^{2}}{2\left(E_{B}-\bar{E}\right)},
$$

где $\bar{E}=\left(E_{+}+E_{-}\right) / 2$. Зависимости $E_{n}\left(E_{0}\right)$ приведены на рис. 2. Видно, что при больших $U_{-}$и малых $U_{+}$максимальная напряженность поля в нейтральной области $n$-слоя значительно превосходит величину $E_{e} \leq 0.1 E_{B}$, при которой зависимость $v(E)$ начинает отличаться от линейной. Из формул (4) и (16) можно получить максимальное значение $\hat{U}_{n}$ падения напряжения на нейтральной части $n$-слоя $U_{n}=E_{n}(d-w)$. Точная формула очень громоздкая, поэтому мы приводим здесь приближенную формулу $\hat{U}_{n}=4 U_{-} / 27$ для случая $U_{+}=0$, которая обеспечивает погрешность менее $15 \%$ и дает точное значение $\hat{U}_{n}$ при $U_{-}=U_{B}$.

В движущейся системе координат уравнение (6) принимает вид

$$
u_{f} \frac{\partial\left(E_{0}-E_{n}\right)}{\partial E_{0}} \frac{\partial E_{0}}{\partial \tilde{z}}+\frac{E_{B}}{d} v\left[E_{n}\left(E_{0}\right)\right]=0
$$




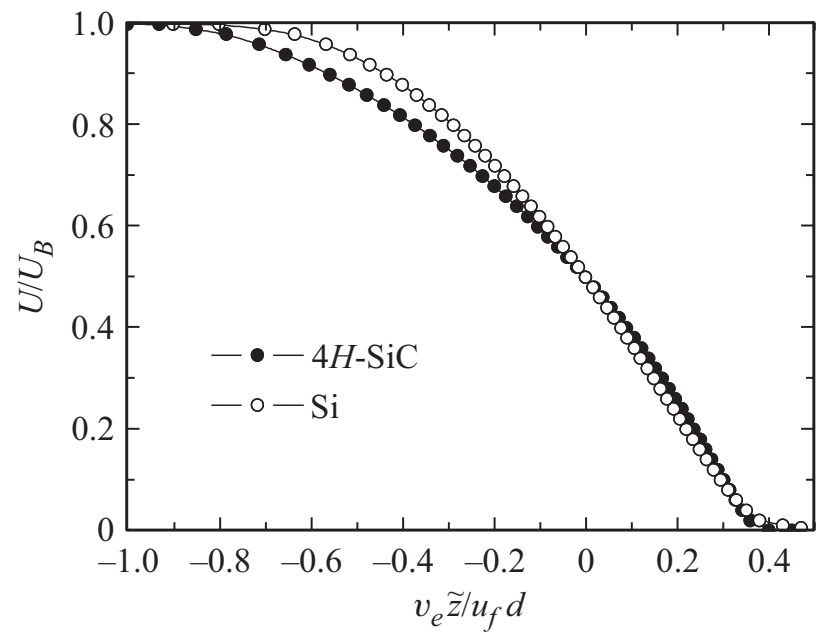

Рис. 3. Распределения напряжения $U$ на фронте ударной волны в кремниевой (светлые символы) и карбид-кремниевой (темные символы) линиях, рассчитанные по формулам (17) и (21) при $U_{-}=U_{B}$ и $U_{+}=0$.

и интегрируется с учетом (16) при любой зависимости $v(E)$ :

$$
\tilde{z}=u_{f} d \int_{E_{0}}^{\bar{E}} \frac{\left(1-\bar{E} / E_{B}\right) d E}{v\left[E_{n}(E)\right] \sqrt{\left(E-E_{B}\right)^{2}+\left(E_{-}-E\right)\left(E-E_{+}\right)}},
$$

начало координат на оси $\tilde{z}$ выбрано в точке, где $E_{0}=\bar{E}$. Примеры распределений напряжения $U$ вдоль линии, рассчитанной по формулам (17), (21), приведены на рис. 3. Как видно, структуры фронта в ЛП на основе $\mathrm{Si}$ и 4H-SiC практически совпадают, несмотря на существенное различие между типами зависимостей $v(E)$ в этих материалах. Если $E_{-}<E_{B}$, то интеграл (21) в расходится логарифмически при $E_{0} \rightarrow E_{ \pm}$, так как при этом $v\left(E_{n}\right) \propto\left|E_{0}-E_{ \pm}\right|$. Поэтому при удалении от фронта $E_{0}$ приближается к $E_{ \pm}$по закону

$$
E_{0}(\tilde{z})-E_{ \pm} \propto \pm \exp (\mp \tilde{z} / \lambda),
$$

где $\lambda=2 u_{f} t_{M} \frac{E_{B}-\bar{E}}{E_{-}-E_{+}}, t_{M}=\varepsilon / e N \mu_{e}-$ время максвелловской релаксации в $n$-области. Вырожденный случай $E_{-}=E_{B}$ отличается тем, что в формуле (22) нужно заменить $\lambda$ на $\lambda / 2$ при $E_{0} \rightarrow E_{-}$. Из (21) следует, что характерное время нарастания напряженности поля на фронте

$$
\begin{aligned}
t_{f} & =\left.\frac{E_{-}-E_{+}}{u_{f}} \frac{d \tilde{z}}{d E_{0}}\right|_{\bar{z}=0} \\
& =\frac{d\left(E_{-}-E_{+}\right)\left(E_{B}-\bar{E}\right)}{E_{B} v\left[E_{n}(\bar{E})\right]} \sqrt{\frac{2}{\left(E_{B}-E_{+}\right)^{2}+\left(E_{B}-E_{-}\right)^{2}}} .
\end{aligned}
$$

Примеры зависимостей $t_{f}$ от $U_{-}$, рассчитанные по этой формуле, приведены на рис. 4. Постоянство $t_{f}$ в $\mathrm{Si}$ и увеличение в $4 H-\mathrm{SiC}$ при больших $U_{-}$обусловлено соответственно насыщением зависимости $v(E)$ и уменьшением $v(E)$ в сильных полях $E_{n}$. Однако характерные скорости нарастания напряжения $U_{-} / t_{f}$ на фронте в обоих случаях монотонно увеличиваются с ростом $U_{-}$ и максимальны при $U_{-}=U_{B}$.

При прохождении фронта ударной волны в нейтральной части $n$-слоя диода рассеивается мощность с линейной плотностью $P_{n}=l E_{n}(d-w) e N v\left(E_{n}\right)$. Используя (4) и (6), эту формулу можно представить в виде

$$
P_{n}=l d \varepsilon E_{n}\left(1-\frac{E_{0}-E_{n}}{E_{B}}\right)\left(1-\frac{\partial E_{n}}{\partial E_{0}}\right) \frac{\partial E_{0}}{\partial t} .
$$

Интегрируя (24) по времени с учетом (16), нетрудно получить линейную плотность энергии, рассеянной на фронте после прохождения волны:

$$
\begin{aligned}
& \omega_{n}=\int_{-\infty}^{\infty} P_{n} d t \\
& =l d \frac{\varepsilon}{E_{B}}\left[\frac{\left(E_{B}-E_{+}\right)^{3}-\left(E_{B}-E_{-}\right)^{3}}{3}-\left(E_{-}-E_{+}\right)\left(E_{B}-\bar{E}\right)^{2}\right] .
\end{aligned}
$$

При $E_{+}=0$ формула (25) упрощается и принимает следующий вид:

$$
\omega_{n}=l d \varepsilon \frac{E_{-}^{3}}{12 E_{B}} .
$$

Как видно, в этом случае $\omega_{n}$ в $2 \sqrt{U_{b} / U_{-}}$раза меньше линейной плотности энергии электрического поля $\omega_{-}=l d \varepsilon E_{-}^{2} / 6$ в ЛП, заряженной до напряжения $U_{-}$.

Следует отметить неожиданное свойство изучаемой ЛП: величина подвижности электронов и характер ее зависимости от напряженности поля определяют толщину фронта $\delta_{f}$ и время нарастания напряжения $t_{f}$

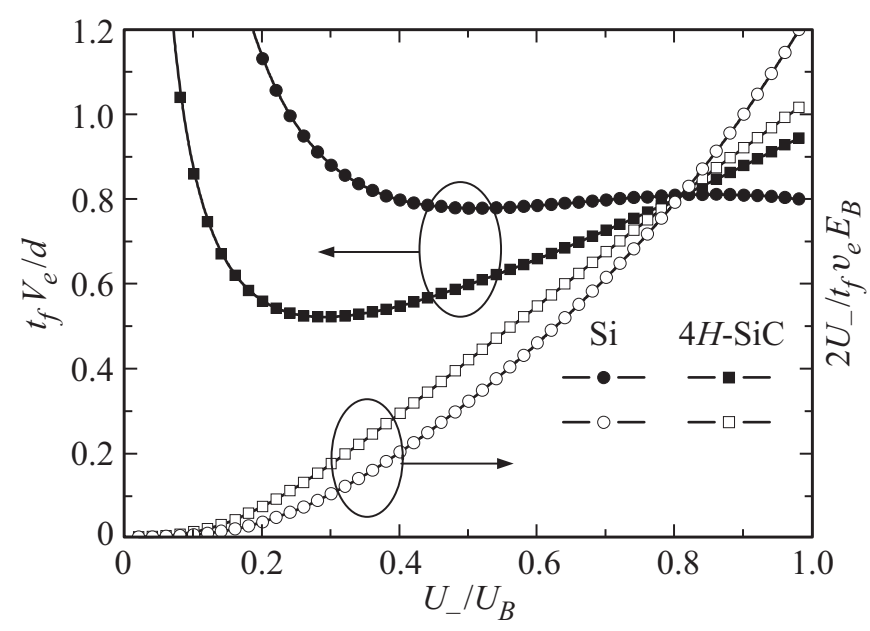

Рис. 4. Зависимости нормированных длительности $t_{f}$ фронта ударной волны (темные символы) и скорости нарастания напряжения $U_{-} / t_{f}$ на фронте (светлые символы) от $U_{-}$, рассчитанные по формуле (23) для кремниевой и карбидкремниевой линий при $U_{+}=0$. 
(см. (21)-(23)), но никак не влияют на максимальное падение напряжения $\hat{U}_{n}$ и линейную плотность энергии потерь $\omega_{n}$ в нейтральной части $n$-слоя.

Наибольший практический интерес представляет случай $E_{+}=0, E_{-}=E_{B}$, когда $^{1} t_{f} \approx \xi d / \nu_{e}$ и толщина фронта $\delta_{f}=u_{f} t_{f} \approx \xi c_{s} d / v_{e} \sqrt{2 \gamma}$, где $\gamma=\left(1+d_{s} / d\right)$. Используя полученные результаты, можно показать, что в этом случае оба условия (13) выполняются при

$$
d_{s} / d \ll \min \left(\frac{N_{s} \mu_{e s}}{N \mu_{e}}, \frac{2 c_{s}}{v_{e}} \sqrt{\xi \frac{N \mu_{e} E_{e}}{N_{s} \mu_{e s} E_{B}}}\right) .
$$

Правая часть этого неравенства максимальна при

$$
\frac{N_{s} \mu_{e x}}{N \mu_{e}}=\left[\xi \frac{E_{e}}{E_{B}}\left(2 \frac{c_{s}}{v_{e}}\right)^{2}\right]^{1 / 3} \equiv \gamma_{0},
$$

откуда следует соотношение

$$
\frac{\delta_{f}}{d+d_{s}} \approx\left(\frac{\gamma_{0}}{\gamma}\right)^{3 / 2} \sqrt{\frac{E_{B}}{8 E_{e}}}
$$

Так как обычно $E_{e}<0.1 E_{B}$, то при условии (27) или, что то же самое, $\gamma \ll \gamma_{0}$ выполняется сильное неравенство $\delta_{f} \gg\left(d+d_{s}\right)$, которое оправдывает применение телеграфных уравнений (1) и одномерных соотношений (3)-(8) для анализа структуры фронта ударной волны.

\section{4. Формирование и затухание ударной волны}

Точный учет временно́й дисперсии и диссипации с помощью формул (3)-(6) и (8) необходим только вблизи фронта, где плотность тока $J$ очень велика и быстро изменяется со временем. При удалении от фронта (при $|\tilde{z}|>\lambda)$ плотность тока $J$ и скорость его изменения экспоненциально уменьшаются. Поэтому в формулах (3), (4) можно положить $E_{n}=E_{s}=0$ и использовать вместо них одно простое соотношение

$$
\frac{U}{U_{B}} \equiv V=\left(\frac{Q}{Q_{B}}\right)^{2}
$$

которое правильно описывает нелинейность емкости диода. Разумеется, при этом не учитываются дисперсия и диссипация на фронте. Кроме того, для упрощения задачи будем считать погонное сопротивление $R_{L}$ постоянной величиной, пренебрегая тем самым скинэффектом в металле электродов. Тогда из (1) следует уравнение

$$
\frac{\partial^{2} q}{\partial \theta^{2}}+(r+g) \frac{\partial q}{\partial \theta}+r g q=\frac{\partial}{\partial \chi}\left(q \frac{\partial q}{\partial \chi}\right),
$$

которое можно использовать для качественного анализа процессов формирования и затухания ударной волны в

\footnotetext{
${ }^{1}$ Множитель $\xi$ близок к единице; он равен 0.8 для $\mathrm{Si}$ и 0.96 для $4 H$-SiC (см. рис. 4).
}

ЛП, на вход которой подается медленно нарастающее напряжение. В (30) использованы безразмерные переменные

$$
q=Q / Q_{B}, \quad \theta=t / t_{0}, \quad \chi=\frac{z}{t_{0}} \sqrt{\frac{L \bar{C}_{B}}{2}},
$$

параметры $r=t_{0} R_{L} / L, g=t_{0} / \tau_{g}$, характеризующие диссипацию за фронтом, и учтено, что в обратно смещенных диодах линейная плотность тока утечки $G U=e N l w / \tau_{g}=Q / \tau_{g}$, где $\tau_{g}=t_{g} N / n_{i}, t_{g}$ - генерационное время жизни, $n_{i}$ - собственная концентрация в полупроводнике, $\bar{C}_{B}=2 \varepsilon l / d$. Далее мы будем использовать простейшие начальное и граничное условия

$$
q(0, \chi)=0 \quad \text { и } \quad q(\theta, 0)=\left\{\begin{array}{ll}
\sqrt{\theta} & \text { при } \theta<1 \\
1 & \text { при } \theta>1
\end{array},\right.
$$

соответствующие линейному нарастанию напряжения на входе в незаряженную ЛП от нуля до $U_{B}$ за время $t_{0}$. Обычно параметр $r g /(r+g)^{2}$, характеризующий относительный вклад в затухание волны двух различных механизмов, очень мал вследствие неравенства $L / R_{L} \ll \tau_{g} \sim 1$ мс, поэтому можно считать $g=0$.

Если еще и сопротивление $R_{L}$ пренебрежимо мало, то нетрудно [3-6,22] найти частное решение задачи Коши (30), (31), которое описывает распространение так называемой простой волны [22], возбуждаемой внешним источником напряжения на входе (т.е. при $z=0$ ) в незаряженную ЛП:

$$
\chi=\sqrt{q}\left(\theta-q^{2}\right) .
$$

Эта формула применима при $\chi_{\theta} \leq \chi \leq \chi_{f}(\theta)$ и $1 \geq q \geq q_{f}(\theta)$, где $\chi_{\theta}=\theta H(\theta-1), H(x)-$ ступенчатая функция Хевисайда, $\chi_{f}$ и $q_{f}$ - положение и амплитуда разрыва, который в этом приближении образуется вместо фронта конечной толщины и устраняет нефизичную двузначность зависимости (32) $q$ от $\chi$ [22]. Если $t_{f} \ll t_{0}$, то сам фронт является квазистационарным, так что для вычисления его структуры и скорости можно использовать соотношения, полученные в предыдущем разделе, полагая $U_{-}=U_{B} q_{f}^{2}$. В частности, безразмерная скорость разрыва $v_{f}=\partial \chi_{f} / \partial \theta$ должна быть равна $\sqrt{q_{f} / 2}$, поэтому дифференцирование (32) по $\theta$ при $q=q_{f}$ приводит к уравнению

$$
\left(\theta-5 q_{f}^{2}\right) \frac{\partial q_{f}}{\partial \theta}=(2-\sqrt{2}) q_{f}
$$

решая которое с начальным условием $q_{f}(0)=0$, получим

$$
\begin{gathered}
q_{f}=\sqrt{\frac{\theta}{\chi_{1}+1}}, \quad \chi_{f}=\chi_{1}\left(\frac{\theta}{\chi_{1}+1}\right)^{5 / 4}, \\
q_{f}=\left(\chi_{f} / \chi_{1}\right)^{2 / 5},
\end{gathered}
$$

где $\chi_{1}=2 \sqrt{2} /(5-2 \sqrt{2}) \approx 1.3$. Формулы (32)-(34) отлично описывают численное решение задачи Коши (30), (31) без учета затухания, приведенное на рис. 5. 


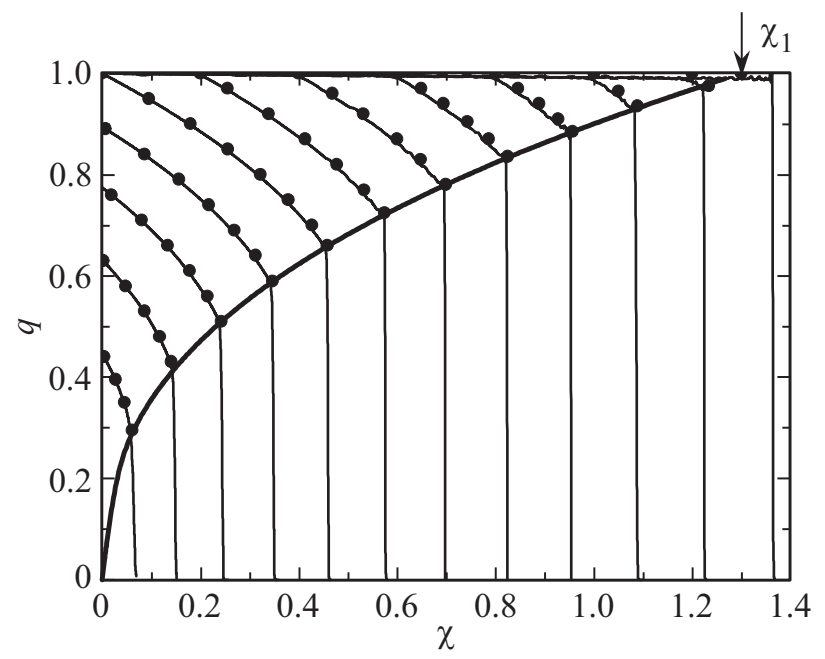

Рис. 5. Профили незатухающей ударной волны в моменты $\theta=0.2 ; 0.4 \ldots 2.4$ при $r=g=0$. Тонкие сплошные линии численное решение задачи Коши (30), (31), темные символы - расчет $q(\theta, \chi)$ по формуле (32), толстая линия зависимость $q_{f}\left(\chi_{f}\right)$, рассчитанная по формуле (34).

При $\theta>1$ появляется область $0<\chi<\chi_{\theta}$, в которой $q=1$, тогда как при $\chi_{\theta}<\chi<\chi_{f}$ профиль волны попрежнему описывается формулой (32). Правая граница $\chi_{\theta}$ движется со скоростью $\partial \chi /\left.\partial \vartheta\right|_{q=1}=1$ и догоняет фронт в плоскости $\chi=\chi_{1}$ в момент $\theta=\theta_{1}=1+\chi_{1}$. После этого возникает стационарная волна с амплитудой $q_{f}=1$ и безразмерной скоростью фронта $v_{f}=\sqrt{1 / 2}$. Используя (26) и (34), нетрудно показать, что за время $\theta_{1}$ в нейтральной части $n$-слоя на фронте рассеивается энергия

$$
\Omega_{1} \equiv \int_{0}^{z_{1}} \omega_{n}\left[q_{f}(z)\right] d z=\frac{5}{66} l z_{1} \varepsilon E_{B} U_{B},
$$

которая в 4.4 раза меньше энергии электрического поля $\omega_{-} z_{1}$, запасенной к этому времени в линии.

При конечном сопротивлении $R_{L}$ аналитическое решение задачи Коши (30), (31) неизвестно, даже если считать $R_{L}=$ const. Результаты численного решения вполне ожидаемы: омические потери в электродах приводят к затуханию волны, как это изображено на рис. 6. Амплитуда скачка напряжения достигает максимума $V_{m}=q_{m}^{2}$ при $\chi=\chi_{m}$ в момент $\theta=\theta_{m}$. Поэтому оптимальная длина ЛП, используемой для обострения фронта входного импульса, равна $z_{m}=\chi_{m} t_{0} \sqrt{2 / L \bar{C}_{B}}$.

Величины $\chi_{m}$ и $\theta_{m}$ очень слабо уменьшаются с ростом $r$ (см. рис. 7) и с достаточной для практических целей точностью могут считаться постоянными и равными $\chi_{1}$ и $\theta_{1}$ соответственно. Максимальная амплитуда разрыва $V_{m}=U_{m} / U_{B}$, естественно, тоже уменьшается с ростом $r$, но медленнее, чем нарастает омическое падение напряжения $U_{r}$ на электродах между входом в линию и плоскостью $\chi=\chi_{m}$, равное в рассматриваемом здесь случае [23]

$$
\begin{aligned}
\frac{U_{r}\left(t_{m}\right)}{U_{B}} & \equiv V_{r}\left(\theta_{m}\right)=1-\left.\frac{1}{r}\left(e^{r}-1\right) e^{-r \theta_{m}}\right|_{t_{m} \ll t_{r}} \\
& \approx 4.6 \frac{t_{0}}{t_{r}}
\end{aligned}
$$

где $t_{r}=\sigma_{M} \mu_{0} d_{M}\left(d+d_{s}\right), \sigma_{M}$ и $d_{M}-$ проводимость и толщина электродов соответственно. Причина этого состоит в том, что затухание приводит к уменьшение со временем тока $I$ вдоль линии, так что второе слагаемое в (1) частично компенсирует „омическое“ поле $R_{L} I$ и замедляет уменьшение напряжения $U$ с ростом $z$.

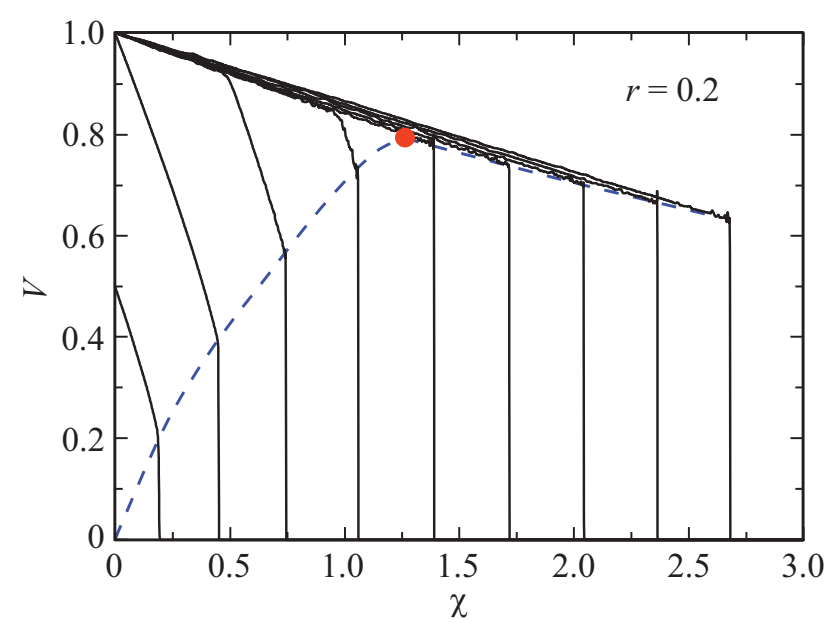

Рис. 6. Сплошные линии - профили затухающей ударной волны в моменты $\theta=0.5 ; 1.0 \ldots 4.5$, полученные путем численного решения задачи Коши (30), (31), при $r=0.2$ и $g=0$. Штриховая линия - зависимость амплитуды разрыва от его положения. Точкой отмечено положение $\chi_{m}$ и амплитуда $V_{m}=q_{m}^{2}$ максимального разрыва в момент $\theta=\theta_{m}=2.3$.

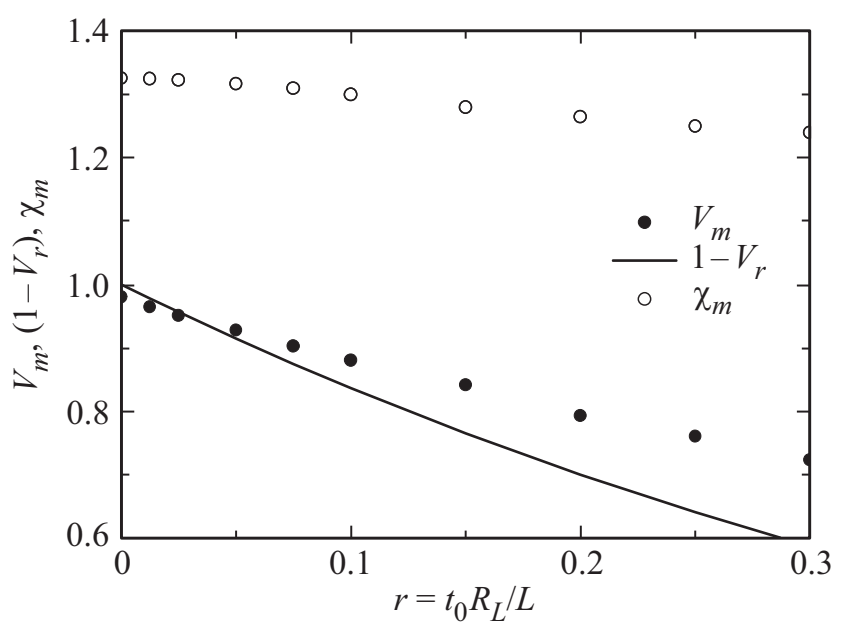

Рис. 7. Зависимости положения $\chi_{m}$ и амплитуды $V_{m}=q_{m}^{2}$ максимального разрыва от безразмерного сопротивления $r$. Символы - результаты численного решения, сплошная линия - расчет $\left(1-V_{r}\right)$ по формуле (35) при $\theta_{m}=2.3$. 
Сопротивление $R_{L}$ уменьшается с ростом $d_{M}$ и $\sigma_{M}$, но ограничено снизу скин-эффектом, учет которого приводит к тому, что вместо (30) получается нелинейное интегро-дифференциальное уравнение. Его решение даже численными методами представляет собой нетривиальную задачу. Однако при сильном скин-эффекте (т. е. при $\left.d_{M}>2 \sqrt{t_{m} / \sigma_{M} \mu_{0}}\right)$ для омического падения напряжения $U_{\sigma}$ вдоль электродов между входом в линию и фронтом была получена точная формула [23]. При малом затухании в электродах, когда $t_{0} \ll t_{\sigma}=\pi \sigma \mu_{0}\left(d+d_{s}\right)^{2}$, ее можно представить в виде

$$
\begin{aligned}
\frac{U_{\sigma}(t)}{U_{B}} & \equiv V_{\sigma}(\theta)=\frac{4}{3} \sqrt{\frac{t_{0}}{t_{\sigma}}}\left[\theta^{3 / 2}-(\theta-1)^{3 / 2} H(\theta-1)\right]_{t=t_{m}} \\
& \approx 2.67 \sqrt{\frac{t_{0}}{t_{\sigma}}}
\end{aligned}
$$

Очевидно, при $V_{\sigma}\left(\theta_{m}\right) \ll 1$ скин-эффект не может привести к изменению соотношений $\chi_{m} \approx \chi_{1}$ и $\theta_{m} \approx 1+\chi_{1}$, использование которых приводит к последнему приближенному равенству в (37).

\section{5. Заключение}

Полученные выше результаты позволяют оценить достижимые параметры ЛП как обострителя фронта импульса напряжения. В качестве примера проделаем это для ЛП на основе $\mathrm{Si}$ и $4 \mathrm{H}-\mathrm{SiC}$, с помощью которых нужно сформировать импульс с фронтом $t_{f}=0.2 \mathrm{Hс}$ и коэффициентом обострения $k=t_{0} / t_{f}=10$. Эти условия позволяют найти все необходимые параметры линии:

- толщину $n$-слоя $d=t_{f} v_{e} / \chi$;

- напряжение пробоя $U_{B}$ и напряженность поля $E_{B}$ по формулам (5);

- величину $\gamma_{0}$ по формуле (28) и полную толщину полупроводника $\gamma d$;

- оптимальную длину линии $z_{m}=\chi_{m} t_{0} c_{s} / \sqrt{\gamma}$;

- минимальную толщину $d_{M}=2 \sqrt{\theta_{m} t_{0} / \sigma_{M} \mu_{0}}$ электродов, при которой их сопротивление определяется скинэфффектом;

- нормированное падение напряжения вдоль электродов $V_{m}=U_{\sigma}\left(\theta_{m} t_{0}\right) / U_{B}$ по формуле $(37)$;

- нормированную ширину линии $l / \gamma d=Z_{s} / Z \sqrt{2 \gamma}$.

Результаты расчетов при $\gamma=\gamma_{0} / 4, \sigma_{M}=3 \cdot 10^{5} \mathrm{CM} / \mathrm{cm}$ и Ом, приведенные в табл. 2, позволяют сделать

Таблица 2. Расчетные параметры ЛП с $Z=5$ Ом на основе $\mathrm{Si}$ и $4 H-\mathrm{SiC}$

\begin{tabular}{c|c|c|c|c|c|c|c|c}
\hline Параметр & $\begin{array}{c}E_{B}, \\
\mathrm{MB} / \mathrm{cM}\end{array}$ & $\begin{array}{c}U_{B}, \\
\text { кВ }\end{array}$ & $\begin{array}{c}d, \\
\text { мКм }\end{array}$ & $\begin{array}{c}\gamma d, \\
\text { МКм }\end{array}$ & $\begin{array}{c}z_{m}, \\
\text { см }\end{array}$ & $\begin{array}{c}d_{M}, \\
\text { мКм }\end{array}$ & $V_{m}$ & $l / \gamma d$ \\
\hline $\mathrm{Si}$ & 0.27 & 0.37 & 27 & 290 & 6.9 & 22 & 0.037 & 4.7 \\
$4 H-\mathrm{SiC}$ & 2.1 & 4.48 & 43 & 240 & 10.6 & 22 & 0.046 & 7.2
\end{tabular}

следующие выводы. Во-первых, необходимая длительность фронта $t_{f}$ однозначно определяет его максимальную амплитуду $U=U_{B}$. По этому показателю карбидкремниевые ЛП обладают явным преимущество над кремниевыми. Причиной этого, очевидно, является гораздо более высокая электрическая прочность карбида кремния. Во-вторых, в рассмотренном случае омическое падение напряжения вдоль электродов $U_{\sigma}$ много меньше $U_{B}$ и даже почти в 3 раза меньше $\hat{U}_{n}$. Поэтому общие потери энергии в ЛП за время $t_{m}$ лишь немного превосходят $\Omega_{1}$. В-третьих, увеличение коэффициента обострения $k$ при заданной длительности фронта приводит к росту минимальной толщины $d_{M}$ электродов, напряжения $U_{\sigma}$ и особенно быстрому росту оптимальной длины линии $z_{m}$, которая и при $k=10$ оказывается очень большой. Вероятно, именно это последнее обстоятельство является основным конструктивным ограничением максимально допустимой величины $k$.

Автор благодарен А.В. Горбатюку и О.В. Руденко за плодотворные обсуждения процессов эволюции нелинейных распределенных систем. Работа выполнена при поддержке РФФИ (грант 16-08-01292).

\section{Список литературы}

[1] R. Landauer. IBM J. Res. Develop., 4, 391 (1960).

[2] Р.В. Хохлов. Радиотехника и электроника, 6, 916 (1961).

[3] И.Г. Катаев. Ударные электромагнитные волны (М., Сов. радио, 1963).

[4] Л.А. Моругин, Г.В. Глебович. Наносекундная импульсная техника (М., Сов. радио, 1964).

[5] А.В. Гапонов, Л.А. Островский, Г.И. Фрейдман. Изв. вузов. Радиофизика, 10, 1378 (1967).

[6] P.W. Smith. Transient Electronics. Pulsed Circuit Technology (Chichester, John Wiley \& Sons Ltd., 2002).

[7] Л.А. Вайнштейн. Электромагнитные волны (М., Радио и связь, 1988).

[8] S.T. Peng, R. Landauer. IBM J. Res. Develop., 17, 299 (1973).

[9] R. Owens, C. Eng, G. White. Proc. IEE, 113, 1763 (1966).

[10] E. Cumberbatch. SIAM J. Appl. Mathematics, 15, 450 (1967).

[11] S.K. Mullick. IBM J. Res. Develop., 11, 558 (1967).

[12] D. Jäger. IEEE Trans. Microwave Theory Techn., 24, 566 (1976).

[13] M.M. Turner, G. Branch, P.W. Smith. IEEE Trans. Electron Dev., 38, 810 (1991).

[14] А.С. Кюрегян, С.Н. Юрков. ФТП, 23, 1819 (1989).

[15] А.С. Кюрегян. ФТП, 50, 293 (2016).

[16] M.V. Fischetti, S.E. Laux. Phys. Rev. B, 38, 9721 (1988).

[17] A. Akturk, N. Goldsman, S. Potbhare, A. Lelis. J. Appl. Phys., 105, 033703 (2009).

[18] H. Guckel, P.A. Brennan, I. Palócz. IEEE Trans. Microwave Theory Techn., 15, 468 (1967).

[19] H. Hasegawa, M. Furukawa, H. Yanai. IEEE Trans. Microwave Theory Techn., 19, 869 (1971).

[20] G.W. Hughes, R.M. White. IEEE Trans. Electron Dev., 22, 945 (1975). 
[21] D.F. Williams. IEEE Trans. Microwave Theory Techn., 47, 176 (1999).

[22] Дж. Уизем. Линейные и нелинейные волны (М., Мир, 1977) [G.B. Whitham. Linear and Nonlinear Waves (John Wiley \& Sons Inc., N.Y., 1974)].

[23] А.С. Кюрегян. Журн. радиоэлектроники [электронный журнал], № 10 (2018). Режим доступа: http://jre.cplire.ru/jre/oct18/9/text.pdf

Редактор Г.А.Оганесян

\title{
Shock electromagnetic wave of large amplitude in the nonlinear trasmission line based on distributed semiconductor diode
}

\section{A.S. Kyuregyan}

All-Russian Electrical Engineering Institute, 111250 Moscow, Russia

\begin{abstract}
An analytical solution to the problem of formation and propagation of large amplitude shock electromagnetic waves in strip transmission line (TL) based on the distributed semiconductor diode was obtained. This solution correctly takes into account nonlinearity, time dispersion and dissipation of TL. The results of the theory has been to estimate the parameters of TL as peaker of the high voltage pulse applied to transmission line input.
\end{abstract}

